\title{
Rastros de violencia institucional: retos para el gerente educativo como constructor de paz en la escuela*
}

Traces of Institutional Violence: Challenges for Educational Managers as Peacebuilders in the School

Vestígios de violência institucional: desafios para o gestor educacional como construtor de paz na escola

\author{
David Arturo Ospina-Ramírez** (iD) orcid.org/0000-0002-1954-2489 \\ José María Mosquera-Ñañez *** (iD orcid.org/0000-0002-7209-2480
}

Para citar este artículo: Ospina-Ramírez, D. y Mosquera-Ñañez, J. (2020). Rastros de violencia institucional: retos para el gerente educativo como constructor de paz en la escuela. Revista Colombiana de Educación, 1/79), 203-222. https//doi.org/10.17227/rce.num79-7504.

\section{(c) $(1) \Theta$}

* Este artículo presenta algunos resultados de la investigación "Análisis de las prácticas y expresiones de la violencia sociocultural en contextos escolares", aprobada con el Acuerdo 063 de 2016, de la Universidad Católica de Manizales.

** Magíster en Educación y Desarrollo Humano. Docente Universidad Católica de Manizales. Diseñador de material lúdico y pedagógico para el Centro de Estudios Avanzados en Niñez y Juventud, Manizales, Colombia. Correo electrónico: david0206ospina@gmail.com

*** Especialista en Gerencia Educativa. Aspirante a Magíster en Educación de la Universidad del Cauca. Actualmente se desempeña en la Institución Educativa Agroambiental la Ceja, Paez- Cauca, Colombia. Correo electrónico: mosqueraj@unicauca.edu.co 


\section{Resumen}

Este artículo visibiliza algunos resultados de una investigación que busca identificar las principales percepciones de violencia en las escuelas, enfocándose en aquellas expresiones que se dan entre docentes, administrativos y directivos de instituciones educativas. La investigación se desarrolló con una metodología cualitativa de tipo descriptivo, mediante un análisis categorial de la información. La investigación fue desarrollada con 42 participantes de la ciudad de Popayán (Colombia), que trabajan en instituciones educativas de diversos tipos y que cursan la Especialización en Gerencia Educativa de la Universidad Católica de Manizales. Este documento se centra en las percepciones de violencia que surgen en las áreas administrativas, académicas y directivas de las instituciones educativas y que se convierten en áreas de influencia directa del gerente educativo como gestor del cambio para la construcción de paz en la escuela.

\section{Palabras clave}

paz; violencia; cultura escolar; personal de la escuela: ambiente educacional

\section{Keywords}

peace; violence; school culture; school personnel; educational environment

\begin{abstract}
This article presents some of the results of a research that seeks to identify the main perceptions of violence in schools, focusing mainly on those expressions found among teachers, administrators, and directors of educational institutions. The research used a qualitative descriptive methodology through a categorical analysis of the information. The research was carried out with 42 participants from the city of Popayán (Colombia), who work at different types of schools and who are pursuing the Specialization in Educational Management program at Universidad Católica de Manizales. This document focuses on the perceptions of violence arising in the administrative, academic and management areas of educational institutions and which become areas of direct influence of educational managers as the promoter of change for peacebuilding at school.
\end{abstract}

\section{Resumo}

Este artigo apresenta alguns resultados de uma pesquisa que busca identificar as principais percepções de violência nas escolas, enfocando aquelas expressões que ocorrem entre professores, administradores e diretores de instituições de ensino. A pesquisa foi desenvolvida com uma metodologia qualitativa do tipo descritiva, através de uma análise categorial da informação. Esta foi realizada com 42 participantes da cidade de Popayan (Colômbia) que trabalham em instituições de ensino de vários tipos e estão fazendo Especialização em Gestão Educacional na Universidade Católica de Manizales. Este documento enfoca as percepções de violência que surgem nas áreas administrativa, acadêmica e diretiva das instituições de ensino e que se tornam áreas de influência direta do gestor educacional como gerente de mudança para a construção de paz na escola.

\section{Palavras-chave}

paz; violência; cultura escolar; pessoal escolar; ambiente educacional 


\section{Introducción}

En este artículo se exponen algunos resultados de la investigación "Análisis correlacional de las prácticas y expresiones de la violencia sociocultural en contextos escolares", de la Universidad Católica de Manizales. También, da a conocer las principales expresiones de violencia que perciben los agentes educativos en la escuela desde una mirada institucional, donde las prácticas de violencia no se presentan solo entre niños y jóvenes, sino también en los actores institucionales, como docentes, directivos, personal administrativo y demás colaboradores de las instituciones educativas. La violencia ha permeado todas las relaciones humanas; por ello, esta investigación busca conocer sus principales expresiones y prácticas, con el fin de darlas a conocer a los gerentes educativos y en general a toda la comunidad; y a su vez, facilitar las transformaciones necesarias para que las escuelas puedan ser territorios de paz. El conflicto forma parte de las relaciones humanas, es su naturaleza, lo cual implica diferentes puntos de vista de los sujetos respecto a situaciones dadas. Sin embargo, determinadas prácticas, dinámicas y expresiones son las que detonan expresiones de violencia en las escuelas, afectando las dinámicas de la comunidad educativa y el bienestar de todos sus agentes.

Las investigaciones sobre violencia en la escuela se han desarrollado principalmente hacia expresiones de violencia entre niños, jóvenes y familias. Sin embargo, como se ha mencionado previamente, la violencia institucional enmarca también aquellas prácticas entre docentes, personal administrativo, directivos y demás agentes de la comunidad educativa.

Reconociendo la importancia que tienen los agentes educativos, ya sea en las prácticas de violencia o en las de construcción de paz, Álvarez-García et al. (2010), realizaron una investigación sobre los conocimientos que poseen docentes para formar para la paz en la escuela:

La experiencia, por sí misma, no es suficiente para responder con eficacia a todas las situaciones educativas que al docente se le plantean cotidianamente. La sociedad cambia, y con él el alumnado, las familias y el propio sistema educativo, dando lugar a nuevos retos para el profesorado y a nuevas exigencias recogidas en la legislación educativa. Y no siempre la respuesta a esos nuevos retos y exigencias la puede encontrar el profesorado en su experiencia previa. Así, por ejemplo, el profesorado en activo tiende a reconocer, tanto más cuanto mayor es su antigüedad como docente, sus limitaciones para atender al alumnado con necesidades educativas especiales, así como la importancia de recibir una mayor formación específica al respecto. (p. 38) 
La educación se le ha atribuido principalmente a los docentes, por ello los estudios e investigaciones en el campo se han orientado a cómo se desarrollan en el aula los niños, jóvenes y docentes; sin embargo, desde la gerencia educativa, se puede comprender la complejidad sistémica de una institución educativa y la importancia de indagar por las prácticas de violencia en las diferentes áreas.

\begin{abstract}
Pasa algo curioso con los fenómenos que tienen que ver con conflictos y la escuela. En muchos casos producen reacciones que van del desasosiego hasta la alarma clara, alarma que a menudo trasciende las paredes de la escuela y toca los medios de comunicación y las conversaciones de sobremesa. En otras ocasiones se encuentran reacciones de minimización de los problemas que implican relaciones negativas, o un clima de convivencia enrarecido, señalando que hay otros problemas más importantes. El primer tipo de reacciones aparece más a menudo cuando los episodios de conflicto suponen conductas violentas, consideradas más graves, o hay adultos implicados en el conflicto, normalmente como objeto de una acción negativa hacia ellos, entonces se habla del aumento de la violencia. (Del Barrio, Barrios, Van der Meulen y Gutiérrez, 2003, pp. 65-66)
\end{abstract}

Los autores evidencian que existen prácticas de conflicto y prácticas de violencia, ambas categorías diferentes, pero que suelen unificarse, ya sea para la sobredimensión de la violencia, o para la disminución de su impacto. Este estudio busca conocer las prácticas de violencia que se generan entre agentes educativos y miembros de la comunidad educativa, identificando los imaginarios de los participantes sobre las expresiones de violencia.

\title{
Tipos de violencia en la escuela
}

Los hechos de violencia vividos y la falta de diálogo han caracterizado la sociedad actual ante la supuesta incapacidad de poder convivir bajo la cultura de la paz; generando una población en conmoción, con la sensación constante de desesperanza y más cuando se observa un sistema educativo con estructuras estereotipadas; por consiguiente, es necesario formar a los estudiantes para propender por la vida y el bien común, bajo los valores de equidad, solidaridad y autonomía, asumiendo un compromiso con la sociedad basado en principios democráticos, con capacidad de escucha y mediador de conflictos por medio de acuerdos y no de violencia. Según Arellano (2007) "Es importante recalcar la imposibilidad de cualquier iniciativa educativa para eliminar los conflictos, por naturaleza, dado que forman parte de todo proceso donde interaccione el ser humano" (p. 33) y no exclusivo de la escuela. Asimismo, es importante resaltar que se encontró 
que el conflicto, como componente positivo, fue el dinamizador de los acuerdos y lo que motivó la construcción de los pactos (Delgado-Salazar y Lara-Salcedo, 2008).

La violencia en los diferentes ámbitos del desempeño del ser humano se ha confundido con los conflictos que se generan en la convivencia y en las emergencias actuales. Sin embargo, la violencia cuenta con características que permiten identificar y diferenciar las prácticas de violencia de otro tipo de expresiones. Parafraseando Alvarez-García et al. (2014), es cualquier acción o conducta que causa un daño intencionado a otros sujetos. Esta definición destaca un factor clave para las expresiones de violencia en la escuela, y es que son acciones con intencionalidades claras, que generan daños en otros actores educativos. Sin embargo, la violencia se puede clasificar en diversos tipos; para esta investigación se empleará el análisis de las violencias realizado por Galtung (1981), donde la violencia se compone de una triada que sustenta las expresiones detonantes directas que quiebran las relaciones humanas:

La violencia directa, física y/o verbal, se hace visible a través del comportamiento. Pero la acción humana no surge de la nada: tiene sus raíces. Dos de ellas son indicativas: la cultura de la violencia (heroica, patriótica, patriarcal, etc.), y la estructura violenta en sí misma por ser demasiado represiva, explotadora o alienante; demasiado estricta o permisiva para la comodidad del pueblo. (p. 3)

A continuación, se aborda el componente teórico de estos tres tipos de violencia: estructural, simbólica (o cultural), y directa, esta última es la más relevante en cualquier contexto, por ser la máxima expresión que visibiliza los problemas de determinada comunidad.

\section{La violencia estructural como eje de acción del gerente educativo}

La violencia estructural forma parte de un sistema social, como lo es la inequidad, la injusticia, la falta de oportunidades de estudio o empleo, el mal gobierno o el abuso de poder. Este tipo de violencia, desde su organización, se conecta al sistema social de un determinado grupo, facilita la injusticia y la desigualdad, y genera detonantes de violencia directa entre sus actores. Para Moyers, Blanco y García, (2016), la violencia estructural puede evidenciarse en instituciones públicas o privadas, permeando sus procesos internos, generando institucionalidad.

Cuando la violencia se instala en una institución, ya sea por el mal manejo del poder, por el estilo de liderazgo o por las condiciones económicas y políticas de la institución, se habla entonces de violencia estructural. Para Merton (1965), una estructura social es un entramado de sistemas 
que vinculan las relaciones humanas, que a su vez son generadoras de dinámicas, actitudes e improntas aceptadas por el grupo humano. Desde este postulado, podría decirse que la estructura a su vez es permeada por las interacciones simbólicas y por los imaginarios de la comunidad que soportan dicha estructura. De manera que la violencia estructural es también soportada y aceptada por la sociedad.

La violencia estructural de una institución se ve influenciada por las condiciones de un contexto más grande, como son las de una región o un país, donde su sistema económico y político afecta de manera directa las expresiones de violencia estructural en la escuela.

La inestabilidad política prolongada, la ingobernabilidad y la crisis económica, en América Latina en los últimos años, reflejan la apertura de un periodo de conflictividad que tiene sus manifestaciones más visibles en un estado de anomia generalizada en el plano tanto social como político, está desembocando en un aumento alarmante de los niveles de violencia en todos los estratos y niveles sociales. (Rodríguez, 2004, p. 5)

El gerente educativo desempeña un rol crucial para el desarrollo de una comunidad, pues es quien conoce las condiciones de la región o del territorio, sus características sociopolíticas y económicas, para la fundamentación de estrategias que mitiguen el impacto de la violencia estructural y que su comunidad se vea impulsada hacia el desarrollo, desde una mirada apreciativa y generativa para la construcción de paz desde la escuela. Parafraseando a Ospina-Ramírez y Ospina-Alvarado (2017), la apreciatividad es una mirada que valora los recursos de los sujetos y del contexto, sin desconocer sus problemáticas, pero dando mayor importancia a dichos recursos para la transformación de determinadas condiciones.

En ese sentido, el gerente educativo es quien puede fortalecer en la comunidad una violencia estructural que se amarre al sistema y que a su vez genere mal manejo del poder, mal manejo de los recursos, inequidad e injusticia, o, bien puede asumir una postura generativa, que se constituya en la creación de posibilidades conjunta con todos los colaboradores, desde una mirada participativa, reconociendo las problemáticas del contexto, pero generando acciones de transformación a las mismas.

\section{La violencia cultural en la escuela}

Para Galtung (1981), la violencia cultural es la que se genera por las características culturales de una comunidad y que es aceptada socialmente como algo correcto, pero que genera desequilibrios, injusticias, inequidades y maltrato, por ejemplo, el machismo, la exclusión por identidad sexual, raza, credo o estrato socioeconómico. El autor expresa: 
Las grandes variantes de la violencia pueden explicarse fácilmente en función de la cultura y estructura: violencia cultural y estructural causan violencia directa, y emplean como instrumentos actores violentos que se rebelan contra las estructuras y esgrimen la cultura para legitimar su uso de la violencia. Obviamente, la paz también debe construirse desde la cultura y la estructura, y no sólo en la mente humana. (Galtung, 1981, p. 6)

De manera que la violencia cultural y la violencia simbólica son invisibles para la sociedad; es decir, en las agendas de gobierno, como se ha visto en el caso colombiano, se da prioridad a la finalización de la violencia directa, que es aquella que implica armas y acciones físicas que afectan y vulneran a los otros, como se evidencia en los acuerdos de paz con los grupos armados. Sin embargo, las condiciones estructurales del país, como la pobreza, la desigualdad y la injusticia social no son abordadas por las agendas de gobierno; a su vez, expresiones de violencia cultural, como la corrupción, tampoco son atendidas por el país.

Este ejemplo puede ser aplicado a las instituciones educativas, donde los agentes educativos buscan dar respuesta a las expresiones de violencia directa de sus estudiantes, sin reconocer que en la institución pueden existir causas directas de esta violencia, como el mal direccionamiento por parte de las directivas, o la violencia cultural arraigada en los docentes, ya sea por cuestiones religiosas o políticas, como la discriminación, el racismo o la exclusión. Como se ha mencionado, las tres violencias están interconectadas y se retroalimentan entre ellas.

A continuación se da a conocer la violencia directa desde los aportes de Galtung (1981) y otros autores que amplían el espectro de esta violencia. Se puede decir que los estudios sobre violencia en general, y de la violencia cultural en particular, han estado basados en el paradigma que privilegia la descripción de las condiciones del sistema social, político y simbólico. Estos estudios parten del análisis y el conocimiento de las condiciones en las que se encuentran los actores con el fin de predecir su eventual caída en la violencia, ya sea como víctimas o victimarios. En estos modelos de interpretación persiste la idea de que existe la posibilidad de hallar un mecanismo elemental que la produce, por ejemplo, la frustración, donde en algunas ocasiones se llega a sugerir una cierta complementariedad entre el análisis de las condiciones sociales y la movilización de ciertos recursos por parte de los actores. Si bien estas perspectivas permiten comprender algunos aspectos de la realidad en torno a la violencia (Arteaga- Botello y Dyjak, 2006).

Para los directivos y altos mandos de cualquier tipo de comunidad, sea educativa o no, resulta más sencillo observar las prácticas de violencia culturales que impulsan a la violencia directa, porque esto permite externalizar la responsabilidad de la violencia y ponerla en cabeza de los niños, 
jóvenes, familias o docentes. Sin embargo, también el gerente educativo puede participar de la violencia cultural desde prácticas de exclusión o, como se verá más adelante, desde el exceso de trabajo, las cargas desmesuradas para los docentes o administrativos, o incluso la falta de liderazgo; factores que pueden ser aceptados por la cultura institucional pero que son impulsores de detonantes más violentos.

\section{La violencia directa como distractor institucional}

Para Gatung (1981), la violencia directa genera impactos sociales contundentes que causan desorden en la comunidad, alteran las potencialidades de las relaciones y las degradan a su mínima expresión de posibilidad. Para Jiménez et al. (2016), "la violencia directa se refiere a la interacción de dos o más personas con cuatro formas de expresión: daño físico, psicológico, acoso y violencia sexual" (p. 82).

Para las escuelas, es imperante trabajar por la erradicación de cualquier tipo de violencia, en el caso de la violencia directa, prácticas como la violencia física o el abuso sexual son claramente atendidas con prioridad. Pero es importante reconocer que el gerente educativo y en general todos los agentes educativos pueden ser víctimas o pueden agenciar prácticas de violencia de tipo físico o psicológico. En este tipo de violencia, el abuso también se considera una práctica de violencia. Desde una mirada institucional, dejando por un momento la violencia en los niños, jóvenes y familias, se entrevé que la violencia directa también puede afectar o ser llevada a cabo por los agentes educativos y colaboradores de la comunidad educativa.

La violencia directa normalmente es atribuida a los comportamientos de los niños o jóvenes, pocas veces se observa la institución a sí misma para indagar por sus propias prácticas de violencia, de manera que las expresiones de violencia directa entre niños y jóvenes, sean insultos, groserías o golpes, pueden convertirse en distractores que no faciliten la reflexión desde las diferentes gestiones directiva, académica, de la comunidad o administrativa de una institución.

\section{El gerente educativo como facilitador del cambio}

La gerencia educativa implica diferentes áreas de actuación, sin embargo, para efectos de este estudio se analizarán aquellos campos cercanos al clima organizacional, que pueden fortalecer o mitigar las prácticas de violencia en la escuela. Valbuena, Morillo, Montiel y Hernández (2015) manifiestan que hay tres componentes desde los cuales se pueden analizar los conflictos en la escuela y proporcionar respuestas eficaces que permitan su transformación: estratégico, organizacional y cultural. 
Si se genera una relación entre estos tres componentes y las tres violencias propuestas por Galtung (1981), se podría decir que tienen relación intrínseca con la violencia cultural y la violencia estructural. Durante años se ha preguntado si en las instituciones educativas es la parte administrativa la que debe estar al servicio de lo académico, o es lo académico lo que debe dar respuesta a las necesidades administrativas de la institución; esto sigue generando fuertes desequilibrios y falta de claridades sobre los roles de los diferentes actores, forjando conflictos institucionales que posteriormente pueden Ilegar a prácticas violentas.

El panorama institucional Ileva a considerar que la gerencia educativa es la mediación entre las gestiones administrativa, de la comunidad, académica o directiva, estableciendo en el gerente educativo las competencias suficientes para dar respuesta a las particularidades del contexto en búsqueda del mejoramiento continuo; es decir, que en la comunidad educativa la rivalidad entre lo administrativo y lo académico no tiene sustento, pues todas las gestiones institucionales trabajan mancomunadamente por los mismos objetivos. Sin embargo, estos no son los únicos conflictos que pueden detonar prácticas violentas estructurales o culturales, como ya se ha mencionado, la falta de liderazgo y de equidad, la corrupción en las instituciones, la falta de oportunidades de desarrollo profesional, el machismo y la discriminación, se convierten en detonantes de violencia mucho más evidentes como el acoso, el maltrato psicológico o incluso físico.

El gerente educativo comprende los factores organizacionales y culturales, desde una postura apreciativa y generativa, suministrando respuestas estratégicas para mitigar las prácticas de violencia institucional, permitiendo la emergencia de nuevas maneras de construir la escuela con un carácter de unidad entre los docentes, directivos, administrativos, colaboradores y comunidad educativa en general.

\section{Construcción de paz en la escuela}

La escuela como territorio de paz implica el recogimiento y apoyo de todas las gestiones institucionales. Para Alvarado et al. (2012), los conflictos en cualquier comunidad forman parte del desarrollo individual y social de los sujetos, pues implican diferentes puntos de vista respecto a determinadas situaciones; sin embargo, los autores también manifiestan la importancia del trabajo en equipo para la transformación de los conflictos en oportunidades de innovación conjunta. Silva et al. (2017) manifiestan la importancia que tienen los agentes educativos en momentos históricos para Colombia, por cuanto la firma de los acuerdos de paz permita dar fin a una de las violencias más dramáticas que ha conocido el país, siendo esta una violencia directa ocasionada por el enfrentamiento de diferentes grupos armados legales e ilegales. 
La paz se constituye en una acción cotidiana que concede a las voces de los actores de una comunidad ser escuchadas, en consecuencia, las relaciones y los conflictos son mediados por el respeto, la compasión y el afecto. Allí, el gerente educativo cumple un papel fundamental como gestor de la paz en la escuela, pues es quien debe motivar transformaciones en las dinámicas relacionales de los docentes, el personal administrativo y todos los miembros de la comunidad educativa. De manera que, el afecto, la apreciabilidad y la postura generativa y estratégica se conviertan en recursos clave para el gerente educativo y para los miembros de la comunidad.

\section{Ruta metodológica}

La investigación es de corte cualitativo de tipo descriptivo, empleando un estudio de caso. Sampieri, Fernández y Baptista (2014) exponen que "en la aproximación cualitativa hay una variedad de concepciones o marcos de interpretación, que guardan un común denominador: todo individuo, grupo o sistema social tiene una manera única de ver el mundo y entender situaciones y eventos" (p. 9). Por tanto, este estudio busca conocer las percepciones que tienen los colaboradores de las instituciones educativas, partiendo de lo que estos actores comprenden y relacionan con la violencia estructural, la violencia simbólica o la violencia cultural.

Según Ortega (2005), los estudios descriptivos se enfocan en la observación de una realidad dada, sin intentar influir en esta. Por ello, este estudio describe las percepciones de violencia de la comunidad educativa, sin intentar influenciar sus percepciones por planteamientos teóricos de la violencia y el conflicto. El proyecto de investigación resalta las percepciones de los participantes desde un análisis categorial, tomando como principales categorías, los tres tipos de violencias descritos por Galtung (1981). El proyecto también emplea el diario de campo para, desde la observación, dar cuenta de las principales percepciones de violencia en la escuela; el diario de campo se empleó como recurso de observación en tres grupos focales: el primero, centrado en la indagación de las prácticas que los docentes perciben como violentas; el segundo, enfocado en la identificación de la percepción que tienen los colaboradores y el personal administrativo, y un tercer grupo focal, orientado al reconocimiento de dichas percepciones, en directivos. Este artículo se centra en las narrativas de 32 docentes, 3 colaboradores administrativos y 7 directivos de instituciones educativas del departamento del Cauca, estudiantes de la especialización en gerencia educativa de la Universidad Católica de Manizales.

La investigación se desarrolló en tres fases: a) el levantamiento de información en fuentes secundarias, la construcción teórica que da sustento al estudio y la construcción de instrumentos de recolección de la 
información; b) la aplicación de los instrumentos, que para este grupo de participantes consistió en un grupo focal, y c) el análisis de la información y la devolución de los resultados por medio de una socialización en clase. En este artículo se trabaja la categoría violencia institucional, asumiendo como subcategorías la violencia estructural, la violencia cultural y la violencia directa en la escuela.

\section{Principales resultados y hallazgos}

\section{Imaginarios sobre violencia}

Los relatos que se presentan en este acápite representan de manera significativa las posturas de la mayoría de los participantes. Se puede reconocer que, para los docentes y gerentes educativos, la violencia está asociada a lo que Galtung (1981) entiende como violencia directa, de manera que atribuyen las expresiones de violencia principalmente a sus estudiantes:

La violencia en las instituciones educativas es toda acción humana dada dentro de la comunidad educativa y que obedece al seno familiar, al trato intrafamiliar, al entorno comunitario y a algunos grupos enlazados con la comercialización de narcóticos. Comienza la desobediencia y el joven no tendrá ningún comportamiento aceptable en ningún ámbito. (Relato de profesor, 42 años)

En este relato se puede reconocer que la mirada del docente está puesta en las acciones que agencian los niños y jóvenes, de manera que la violencia en la escuela se asocia únicamente con la violencia directa o sus representaciones; sin embargo, se desconocen la violencia estructural y la violencia cultural que la sustentan.

La violencia son acciones de carácter psicológico como el matoneo, aunque no es muy frecuente en mi escuela porque estamos con una población estudiantil proveniente de la zona rural. Como todo establecimiento educativo se presenta alguna agresión psicológica y física, y se ve en las familias violencia social que se vive en el municipio. (Relato de coordinador de convivencia y profesor, 48 años)

Desde la institucionalidad, los docentes y directivos atribuyen la violencia a factores sociales y culturales, nuevamente entregando la responsabilidad de los actos violentos a los estudiantes y a sus familias. Durante el desarrollo del trabajo de campo, se pudo ver que cuando a los participantes se les preguntó "¿Qué expresiones de violencia se dan en sus instituciones educativas?", en su mayoría, las respuestas estuvieron orientadas a describir los actos violentos que se dan entre los estudiantes; 
por ello el instrumento, que consistió en un grupo focal, permitió abordar las preguntas y buscar otras prácticas que se dan a nivel docente, administrativo y directivo.

\section{Entre la violencia estructural y la violencia cultural}

En algunos casos los participantes manifestaron que sus instituciones educativas no cuentan con los recursos necesarios para dar respuesta a todos los procesos institucionales. Esto se puede asociar con condiciones estructurales de la institución, pero que son aceptadas culturalmente.

Hay cosas de carácter administrativo, como el exceso de trabajo; yo considero que es algo de violencia porque afecta mi paz, mi tranquilidad e incluso mi vida personal. $\mathrm{O}$ a veces dejan trabajo que no es de la competencia del funcionario o no ha sido capacitado, pero si no sale bien debe asumir las consecuencias. Y cuando los compañeros ven que a uno le dejan trabajo desmedido, prefieren guardar silencio. (Relato de profesora, 37 años)

Las condiciones administrativas de la institución afectan las dinámicas de toda la institución y se ven reflejadas en la calidad de la educación. Los docentes perciben la sobrecarga de trabajo como una expresión de violencia porque afecta sus vidas personales y tiempos para las demás áreas de su vida, ya sea el estudio, la familia o cualquier otro tipo de actividad.

La violencia entre docentes se da cuando empezamos a generar chismes, "que llegó tarde el compañero, que no prepara clase, que el compañero se duerme" [...]; contar a otro compañero. Nunca le decimos al directamente afectado, pero sí podemos dañar en cierta forma la armonía en el ambiente porque son cosas que generalmente no se quedan en algo pequeño, sino que se genera un gran chisme y cuando se hace una reunión todo el mundo llega a atacar a ese profesor y él no sabe el motivo. Esto termina por afectarnos a todos. (Relato de profesora y coordinadora académica, 29 años)

Los docentes interpretan los comentarios negativos sobre el desempeño de otros docentes, como aportes que no ayudan al mejoramiento profesional, pero sí constituyen expresiones de violencia. De igual modo, también expresan que este tipo de prácticas son aceptadas por la comunidad educativa en general. En la escuela se evidencian las mezclas y relaciones que existen entre las tres violencias, de manera que para los docentes la violencia estructural se convierte en violencia cultural cuando las condiciones del sistema institucional fortalecen las prácticas de exclusión o la insana competencia entre docentes y otros colaboradores. 


\section{La violencia psicológica como principal expresión de la violencia directa en la escuela}

La falta de motivación y la ausencia de claridades respecto al desarrollo profesional dentro de una institución educativa se asocian con la falta de equidad, injusticia y como principal causa de la desmotivación en los docentes:

En el nivel en el que yo me desenvuelvo, no he experimentado una violencia física, pero sí una violencia psicológica en varios aspectos: No se maneja recompensa laboral o reconocimiento, solo se llama la atención a lo que se hace mal. Se viven amiguismos en la institución, entonces se reconoce es al "amigo" y no al que hace bien su trabajo; eso genera un desbalance enorme y una gran desmotivación. (Profesora y decana, 32 años)

También se considera violencia psicológica la constante batalla de conocimientos que se da entre docentes, descalificando el conocimiento de otros y donde se generan competencias profesionales continuamente.

Entre el docente más bueno sea, más trabajo le van a cargar, a los que no los dejan seguir normal. Los mejores terminan estallando y se van a otra institución. No existe motivación, no se les exalta por la labor. Hay también falta de apoyo por los directivos frente a los docentes que tienen ideas proyectos que quieren hacer parte de la investigación y no se apoya. (Profesora, 35 años)

Para los participantes, la falta de motivación es una expresión de violencia que surge desde los directivos hacia los docentes, pues no les permite desplegar sus capacidades y crear nuevas posibilidades propias y colectivas. Es importante resaltar que los participantes no se refirieron en el trabajo de campo a incentivos económicos, lo que evidencia que el gerente educativo sí es responsable del bienestar institucional en otras áreas que les generan mayor motivación, como poder investigar o poder crear proyectos o participar de otras áreas institucionales.

En el sector de primaria estigmatizan a los docentes, se crean como niveles, entonces los profesores de bachillerato son superiores a los de primaria, creen que los demás no pueden, que no tienen capacidades. Entonces así un profesor quiera experimentar otros campos, la institución y los mismos compañeros le obligan a siempre quedarse en lo mismo. Y por lo general los directivos toman decisiones es con sus compinches o con sus compadres ${ }^{1}$, pero no tienen en cuenta a todo el colectivo docente. (Profesor, 49 años)

1 En Colombia se usan estos términos para referirse a amigos muy cercanos. 
Para los docentes la falta de claridad en el desarrollo profesional y las posibilidades de crecimiento dentro de la institución, permiten inconformidades y lo consideran como parte de la violencia estructural que genera otro tipo de violencias entre compañeros.

\section{Prácticas de violencia docente: la guerra por el conocimiento}

En el trabajo de campo se evidenció que, aunque no existen expresiones claras de violencia, sí existen prácticas que dan cuenta de ambientes hostiles que se generan alrededor de los círculos de conocimiento entre docentes, directivos y comunidad en general:

Creo que la violencia que se genera en la parte de docentes es porque todos quieren tener la razón y el conocimiento verdadero, uno ve que generan expresiones de descalificación a los otros, ya sea en reuniones o en el aula y esto genera posteriores conflictos más personales. (Profesora y decana, 32 años)

Referente a la violencia, los celos profesionales generan en ocasiones formas de violencia. Los directivos o incluso compañeros de trabajo tampoco soportan que los que están por debajo o las personas nuevas tengan la iniciativa entonces muchas veces cierran todas las posibilidades de tener iniciativas. (Relato de profesora, 38 años)

En estos dos relatos se evidencia que, en las instituciones educativas, de manera particular y como factor diferencial de otro tipo de instituciones, el conocimiento es el factor clave para el desarrollo profesional y colectivo, pero en los círculos de conocimiento se fomentan las competencias malsanas que no se fortalecen desde una mirada crítica y apreciativa, sino que generan imposibilidades de crecimiento para los docentes y para la institución educativa en general.

Como se ha mencionado antes, la escuela como lugar de encuentros y desencuentros, y sin negar el conflicto, acoge la diferencia del ser humano y genera propuestas emancipadoras que se enmarcan en una concepción positiva del conflicto, y del mismo modo dinamizar las relaciones entre los estudiantes y profesores, en el marco de valores como la equidad, el respeto, el bien común, la libertad, la justicia, el diálogo y la paz (Delgado-Salazar y Lara-Salcedo, 2008).

\section{Miradas en conflicto: entre lo académico y lo administrativo}

En las instituciones educativas existen cuatro tipos de gestiones propuestas por el MEN (2008): directiva, académica, administrativa y comunitaria. En este artículo se tienen en cuenta los estudios de violencia en la escuela que se han centrado principalmente en la violencia entre estudiantes, 
familias o comunidad en general. Los participantes expresaron que también existen conflictos entre las áreas administrativas y las áreas académicas de las instituciones:

Entre docentes y administrativos también se ve violencia, muchas veces los docenes no comprenden que el administrativo quiere ayudar y piensan que lo que hace el administrativo es una pérdida de tiempo, que la institución debe estar volcada a atender solo las necesidades de los docentes. No valoran los esfuerzos de los administrativos, empiezan: “¡Uy!, qué pereza, ya llegaron los psicólogos” o "¡Ay no!, qué mamera más trámites y formatos, esto lo deben hacer es las secretarias". (Relato de profesora, 30 años)

La falta de conocimiento sobre las labores que el otro desempeña parece ser una de las principales causas de expresiones de violencia entre administrativos y docentes, generando imaginarios de poco valor sobre el trabajo de los otros.

Creo que sin la gestión administrativa no habría estudiantes, no se llevarían a cabo las matrículas y en general todos los procesos que requieren del trabajo de los administrativos, efectivamente no se reconoce bien su trabajo, esto genera que los administrativos a veces también sean hostiles con nosotros los docentes, es entendible. (Relato de coordinador de convivencia y profesor, 27 años)

Los procesos institucionales, aunque son reconocidos por los docentes y directivos, no siempre poseen un valor significativo para el correcto funcionamiento de la institución, y como lo menciona este relato, el poco valor que se le da al trabajo de los administrativos, ocasiona tratos hostiles. Desde los anteriores hallazgos, es posible comprender diferentes maneras en que se manifiesta la violencia en la escuela.

\section{Conclusiones y comentarios finales}

Durante la investigación se identificaron diferentes situaciones que los docentes perciben como prácticas de violencia, que tienen relación con la violencia estructural, la violencia cultural y la violencia directa; sin embargo, se evidenció que la violencia está instalada en las instituciones desde la cultura institucional y desde su clima organizacional. Es importante resaltar que en un principio, los participantes asociaron las prácticas de violencia en la escuela, únicamente con el comportamiento de niños, jóvenes y sus familias; fue necesario preguntar específicamente si entre docentes, administrativos y directivos no existían prácticas de violencia;

2 "Mamera" es una expresión colombiana que se usa para expresar que algo es agotador, que es innecesario y que genera pereza su realización. 
aunque no se puede generalizar este resultado, sí se hace evidente la necesidad de invitar a los docentes, directivos, administrativos y todos los colaboradores de una institución educativa, a intervenir sobre sus propias prácticas de violencia.

No se desconoce que factores estructurales, como el poder mal ejercido, la falta de liderazgo o la falta de planificación en las actividades académicas, son generadores de otras violencias en la escuela, pero en los relatos se hizo hincapié en la cultura de las organizaciones como generadora de la violencia entre sus docentes, directivos y colaboradores. Algunos de los participantes atribuyen la violencia cultural al equipo de colaboradores de la escuela, a la falta de claridad en las políticas institucionales para los ascensos, procesos de escalafón docente y falta de comunicación entre los procesos administrativos y académicos e investigativos. Así, los docentes consideran que la academia está al servicio de los fines administrativos institucionales, y no, lo administrativo al servicio de la academia.

Un factor importante es la competencia entre docentes, percibida por los participantes como una expresión de violencia cultural, porque se cree que esta conducta competitiva forma parte de los círculos de conocimiento, pero se considera violenta dado que, desde allí, empieza el desconocimiento de la labor del otro y las críticas destructivas en los equipos de trabajo. De igual forma, los participantes manifiestan que el desconocimiento sobre la importancia que tiene la labor administrativa dentro de la institución, genera tratos hostiles entre docentes y personal administrativo. Desde la gestión directiva de la institución, la falta de claridades, la corrupción institucional y la toma de decisiones sin criterios justos para el desarrollo de toda la comunidad educativa, genera percepciones de violencia en la escuela, con mayor orientación a la violencia estructural. Por otra parte, los docentes manifiestan que la presión laboral, los horarios inadecuados de trabajo y la falta de consideración de los tiempos libres de los docentes por parte de los directivos, genera desmotivación y genera dificultades en la construcción de relaciones laborales.

Es importante reconocer que el gerente educativo tiene un fuerte Ilamado institucional a indagar y transformar las prácticas de violencia que se presentan en su escuela, y a diferenciarlas; por ejemplo, en esta investigación fue necesario categorizar las percepciones de violencia, para identificar aquellos conocimientos generalizados y que se pudieron ubicar en la violencia estructural, la violencia directa o la violencia cultural, dejando de lado las percepciones individuales y no generalizadas que podían ser problemas de tipo personal. Dichas transformaciones requieren de una mirada diferente de los conflictos, pues este es inherente a las relaciones humanas, mientras que la violencia es una elección de los sujetos que intervienen. Para Caballero (2000), la transformación de los conflictos de 
manera constructiva mejora los índices de productividad y eficiencia en la escuela. Por consiguiente, ya sea desde el punto de vista humano o desde el punto de vista estratégico y gerencial, siempre es provechoso para el gerente educativo y para la institución tomar posturas apreciativas frente a los conflictos y facilitar estrategias de transformación de los conflictos en posibilidades de crecimiento conjunto.

Subirana y Cooperrider (2013) proponen que para que exista una transformación de los sujetos y de las organizaciones, es necesario que el líder de la institución o del colectivo tenga una mirada apreciativa sobre el ser humano. Lo anterior permite al gerente educativo externalizar la violencia y verla como una práctica que ni es inherente al ser humano, sino que puede ser aprehendida y por ende modificada; a su vez, le permite internalizar las potencias de los sujetos, de manera que les reconoce sus capacidades y potencialidades para la transformación.

Para Galtung (1998), cuando una comunidad ha sido permeada por la violencia, es necesario trabajar en la reconstrucción, en la reconciliación y en la resolución. Comprendiendo que las violencias propuestas por este autor no siempre son visibles, es necesario que constantemente se trabaje por la construcción de la escuela, pues pueden existir prácticas de violencia que se instalan sin hacerse visibles para la comunidad, o aunque se hagan evidentes, forman parte de la aceptación cultural.

Entonces, para el gerente educativo es fundamental trabajar por la reconstrucción de relaciones y de caminos claros de crecimiento institucional, la reconciliación de los agentes educativos que se han visto afectados o que han sido generadores de prácticas de violencia, y en la resolución de los conflictos que se presentan continuamente en las relaciones humanas. Desde la gestión institucional, considerar las voces de todos es importante para poder favorecer el desarrollo institucional de manera colectiva y sostenible en el tiempo, como lo mencionan Collazos y Ospina-Ramírez (2017), quienes plantean que las comunidades que crecen de manera colaborativa logran tener un impacto positivo y sostenible en la sociedad.

El clima organizacional es un factor importante a la hora de considerar políticas de construcción de paz en la escuela, en palabras de Ospina-Ramírez, Burgos y Madera (2017) el clima organizacional tiene un importante rol en el mejoramiento de las prácticas educativas o en su deterioro, y es labor del gerente de la educación construir colaborativamente un clima organizacional dinámico, activo, generativo y abierto al cambio, que considere los potenciales y recursos de toda la comunidad educativa.

A nivel institucional, es importante considerar si la estructura y las políticas de desarrollo profesional e incentivos en las instituciones, afectan positiva o negativamente el clima organizacional, permitiendo el trabajo colectivo por el alcance de metas y logros misionales, o, por el contrario,

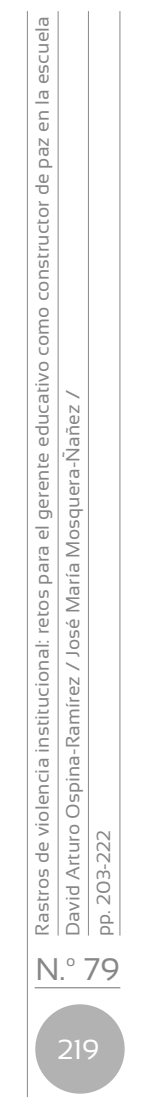


las políticas son generadoras de competencia individual y desarticulada, generando dinámicas organizacionales difíciles de abordar. Para futuros investigadores en el campo, se sugiere considerar que las percepciones de violencia de los docentes y el personal administrativo de las instituciones pueden generar impactos en el desarrollo académico y vivencial de los estudiantes, pues el clima organizacional tiene relación con los potenciales de desarrollo académico de los estudiantes.

\section{Referencias}

Alvarado, S. V., Ospina, H. F., Mejía, M. Q., Luna, M. T., Ospina-Alvarado, M. C. y Patiño, J. A. (2012). Las escuelas como territorios de paz. Buenos Aires: Clacso. http://biblioteca.clacso.edu.ar/clacso/posgrados/20120910105957/LasEscuelascomoTerritoriosdePaz.pdf

Álvarez-García, D., Dobarro, A., Álvarez, L., Núñez, J. C. y Rodríguez, C. (2014). La violencia escolar en los centros de educación secundaria de Asturias desde la perspectiva del alumnado. Educación XX1, 17(2), 337-360. http://www.redalyc.org/ pdf/706/70630580014.pdf

Álvarez-García, D., Rodríguez, C., González-Castro, P., Núñez, J. C. y Álvarez, L. (2010). La formación de los futuros docentes frente a la violencia escolar. Revista de Psicodidáctica, 15(1), 35-56.

Arellano, N. (2007). La violencia escolar y la prevención del conflicto. Orbis. Revista Científica Ciencias Humanas, 3(7), 23-45.

Arteaga Botello, N. y Dyjak Montes de Oca, C. (2006). Las fronteras de la violencia cultural: del estigma tolerable al estigma intolerable. Convergencia, 13(41), 65-86. http://www.scielo.org.mx/scielo.php?script=sci_arttext\&pid=S1405-14352006000200003

Caballero, A. (2000). Transformar los conflictos: una apuesta. Tarbiya, Revista de Investigación e Innovación Educativa, 25, 95-106. https://revistas.uam.es/tarbiya/article/view/7128/7493

Collazos, N. J. y Ospina-Ramírez, D. A. (2017). El desarrollo endógeno como camino para la innovación educativa en zonas rurales. Revista Plumilla, 19, 13-26. http://revistasum.umanizales.edu.co/ojs/index. php/plumillaeducativa/article/view/2471

Del Barrio, C., Barrios, A., Van der Meulen, K., y Gutiérrez, H. (2003). Las distintas perspectivas de estudiantes y docentes acerca de la violencia escolar. Estudios de Juventud 62, 65-79.

Delgado-Salazar, R. y Lara-Salcedo, L. (2008). De la mediación del conflicto escolar a la construcción de comunidades justas. Universitas Psychologica, 7(3), 673-690. 
Galtung, J. (1981). Contribución específica de la irenología al estudio de la violencia: tipologías. En La violencia y sus causas (pp. 91-106). París: UNESCO.

Galtung, J. (1998). Tras la violencia: reconstrucción, reconciliación, resolución. Bilbao: Santa María.

Jiménez, R. M. G., Suárez, C., Polanco, M. M., Hernández, A., Keyser, U., Robles, A. L. y Gutiérrez, E. (2016). ¿Cómo entendemos la violencia de género en las instituciones de educación superior? Marco conceptual. GénEros, 19(11), 79-96. http://bvirtual.ucol.mx/descargables/198_como_entendemos_violencia.pdf

Merton, R. K. (1965). Teoría y estructura social. México D.F.: Fondo de Cultura Económica.

Ministerio de Educación Nacional (MEN) (2008). Guía para el mejoramiento institucional. De la autoevaluación al Plan de Mejoramiento. No. 34. Bogotá.

Palafox, C. G., Espejel, J. E. y Valenzuela, J. Á. (2017). Confianza institucional y violencia estructural en Nogales, Sonora. Región y sociedad, 29(spe5), 215-248. https://dx.doi.org/10.22198/rys.2017.0.a230

Ortega, R. (2005). Violencia escolar en Nicaragua. Un estudio descriptivo en escuelas de primaria. Revista Mexicana de Investigación Educativa, 10(26), 787-804. https://idus.us.es/xmlui/bitstream/ handle/11441/23195/violencia\%20escolar\%20en\%20nicaragua.pd$\mathrm{f}$ ? sequence $=2$

Ospina-Ramírez, D. A., Burgos, S. B. y Madera, J. A. (2017). La gerencia educativa y la gestión del cambio. Revista Diálogos de Saberes, 46, 187-200. Bogotá: Universidad Libre.

Ospina-Ramírez, D. A. y Ospina-Alvarado, M. C. (2017). Futuros posibles, el potencial creativo de niñas y niños para la construcción de paz. Revista Latinoamericana de Ciencias Sociales, Niñez y Juventud, 15(1), 175-192.

Rodríguez, F. (2004). La pobreza como un proceso de violencia estructural. Revista de Ciencias Sociales, 10(1), 42-50. https://www.flacsoandes.edu. ec/sites/default/files/agora/files/1278089538.francisco_rodriguez.pdf

Sampieri, R., Fernández, C. y Baptista, M. (2014). Metodología de la investigación. México: McGraw-Hill, Interamericana Editores S.A.

Silva, G. A., Bermeo, D. B., García, E. B., Perdomo, Y. G. y Martín, J. I. (2017). Otra escuela es posible: subjetividades políticas y retos en el posacuerdo. Revista Educación y Ciudad, (31), 185-193. https://dialnet.unirioja.es/servlet/articulo?codigo $=5803809$

Subirana, M. y Cooperrider, D. (2013). Indagación apreciativa: un enfoque innovador para la transformación personal y de las organizaciones. Barcelona: Editorial Kairós. 
Valbuena, M., Morillo, R., Montiel, M. y Hernández, J. (2015). Gerencia estratégica y los conflictos organizacionales. Multiciencias, 12, 270276. http://www.produccioncientificaluz.org/index.php/multiciencias/article/view/19163 